\title{
Laparoscopic exposure and electrostimulation of the somatic and autonomous pelvic nerves: a new method for implantation of neuroprothesis in paralyzed patients?
}

Published online: 26 February 2004

(C) Springer-Verlag Berlin / Heidelberg 2004

\begin{abstract}
Objective: The purpose of this study was to demonstrate the feasibility of the laparoscopic approach to expose the pelvic autonomous and somatic nerves for possible implantation of neuroprothesis for restoration of locomotion, micturition, defecation and ejaculation in paralyzed patients. Methods: In 146 patients undergoing laparoscopic para-aortic and pelvic lymphadenectomy for cervical cancer, sympathetic, parasympathetic and somatic nerves were precisely exposed in the para-aortic region and in the pelvis, which then allowed electrostimulation of the different nerves. In order to evaluate the motor activity of the stimulated nerves, transurethral and transrectal pressure catheters were employed. Results: Systematic laparoscopic exposure of all somatic and autonomous pelvic nerves was carried out in all 146 consecutive patients without any complications. Intraoperative neurostimulation demonstrated the exact function of the different exposed autonomic nerves. Conclusions: Selective laparoscopic implantation of neuroelectrodes to the somatic and autonomic pelvic nerves in a paralyzed patient may be a feasible and reproducible procedure for surgeons well-trained in laparoscopic surgery.
\end{abstract}

Keywords Laparoscopic neurostimulation - Sciatic nerve $\cdot$ Autonomous nerves $\cdot$ Paraplegic

\section{Introduction}

Preservation of body function and body image is becoming more important for patients and is likely to change surgical management in gynecology. Surgical techniques

No financial support was received for this study, and there was no conflict of interest.

M. Possover $(\varpi)$

Department of Obstetrics and Gynecology,

University of Cologne,

Kerpener Strasse 34, 50931 Cologne, Germany

e-mail: Marc.Possover@medizin.uni-koeln.de

Tel.: +49-0221-4785829

Fax: +49-0221-6789 that preserve the pelvic nerve bundles during a radical hysterectomy for cervical cancer recently have been reported to preserve bladder and rectal function $[1,2,3]$. One obstacle in sparing intrapelvic nerve structures during surgery is their difficult identification and often limited knowledge of the functional anatomy of the pelvis. This study provides strong evidence that laparoscopy offers optimal access for identification of the nerve structures within the retroperitoneal space. Thus, the issue was: (1) to assess laparoscopic surgery for identification and dissection of the somatic and autonomous pelvic nerves in view of laparoscopic implantation of neuroelectrodes in para/tetraplegic patients, (2) to establish a new method for laparoscopic assessment of exposed pelvic nerves and (3) to establish an anatomical correlation between topography and function

\section{Materials and methods}

During the last 6 years, we have performed more than 500 laparoscopic para-aortic and pelvic lymphadenectomies for gynecological malignancies. Our technique of laparoscopic para-aortic and pelvic lymphadenectomy in women with cervical cancer was applied as described elsewhere [4]. During all these procedures, special attention was paid to the exposure of different pelvic nerves. Since we developed the nerve-sparing modification of laparoscopic-assisted radical vaginal hysterectomy for cervical cancer [1], systematic laparoscopic exposure of different pelvic nerves during lymphadenectomy has been performed. The superior hypogastric plexus should be identified as an intricate meshwork of nerve fibers embedded in the connective/fat tissue of the mesocolon dorsal of the inferior mesenteric artery. At the level of the aortic bifurcation, the plexus diverges into both inferior hypogastric nerves, curving outwards, downwards and backwards into the mesosigmoid, ventral to Waldeyer's fascia. The left sympathetic trunk is easily exposed lateral to the aorta, while the right trunk is covered by the inferior cave vein. By transfixing the sacral hypogastric fascia dorsal to the cardinal "ligament" and caudal to the medial rectal artery, the splanchnic pelvic nerves should be exposed. More laterally, by dividing the fibers of the isciococcygeal muscle, the ischiorectal fossa is opened and the pudendal nerve or its branches can be identified.

By freeing the vascular part of the cardinal ligament, exposure of the sacral plexus as well as the sciatic nerve is performed. 
Special exposure of the gluteal nerves is obtained by dissection of the sciatic nerve through the great sciatic foramen.

For intraoperative urodynamic testing a microtip rectal probe and an $8 \mathrm{~F}$ dual sensor microtip transurethral catheter (Unisensor) without filling channel were used. The sensor at the tip of the rectal probe was placed $7 \mathrm{~cm}$ proximal to the anal sphincter. The transurethral catheter was inserted in such a manner that the urethral sensor could be positioned $2 \mathrm{~cm}$ proximal to the external urethral orifice. One perineal self-sticking jelly surface pad electrode was placed lateral to the midpoint between the anus and the urethral orifice on either side; the reference electrode was positioned laterally, near the iliac crest. Bladder filling and emptying during the procedure was regulated with a $12 \mathrm{~F}$ suprapubic catheter. The bladder capacity during nerve stimulation was 150 to $200 \mathrm{cc}$. The urodynamic apparatus used was a portable urodynamic system Libra mobil (Medical Measurement Systems, Enschede, Germany). Whenever nerve stimulation was performed, all three pressure channels were calibrated to zero in order to minimize artifacts caused by the loss or change of intraabdominal pressure during the laparoscopic procedure. Concerning the electrostimulation of the somatic nerve, macroscopic observation of the lower limb was performed to visually confirm a muscle contraction or a motion from a part of the leg.

After the exposure of all of these nerves, laparoscopic neurostimulation was applied with a bipolar or tripolar probe in order to apply a well-defined current to distinct neural structures. The bipolar probe allowed stimulation with 0 to $6 \mathrm{~V}$ and $2.5 \mathrm{~Hz}$, while the development of a tripolar probe (A:D: Krauth $\mathrm{GmbH} \& \mathrm{Co}$, Hamburg) allows stimulation with 3 or $30 \mathrm{~Hz}$ and 3 to $30 \mathrm{~V}$.

Consent was obtained for intraoperative nerve stimulation from the patient as well as from the Ethics Committee at our university.

\section{Results}

Here, we report for the first time about the laparoscopic neurostimulation of 146 consecutive patients who underwent a nerve-sparing laparoscopic-assisted radical vaginal hysterectomy type 3 for cervical cancer. In all cases, the bloodlessness of the procedure allowed an optimal laparoscopic neurostimulation without being influenced by fluid accumulation in the pelvis, and no complications occurred while dissecting the different nerves. During the laparoscopic procedure urodynamic testing was performed without problems of interference from the pneumoperitoneum or instrumentation through the port systems. Exchange of instruments decreased intravesical and rectal pressure by less than $9 \mathrm{~cm}$ of water and did not affect urethral pressure. Urodynamic recordings during the use of the diathermy in the surgical procedure were impossible to interpret: The current was transmitted to other structures causing stimulation that could not be applied selectively. Application of the electrical current using the bipolar probe was easily applied by introducing it through a 5-mm trocar, while the handling of the tripolar probe was more complicated because of its thicker shaft (a 10-mm trocar is reqired) and the need to touch the structure to be stimulated at all three electrode sites. The recordings of the urethral, rectal and intravesical pressures were easily obtained using our setup, and their amplitudes showed good response to changes of the voltage applied to the structure. While electrostimulation of autonomic nerves induced measurable pressure changes even under relaxation, the stimulation of somatic

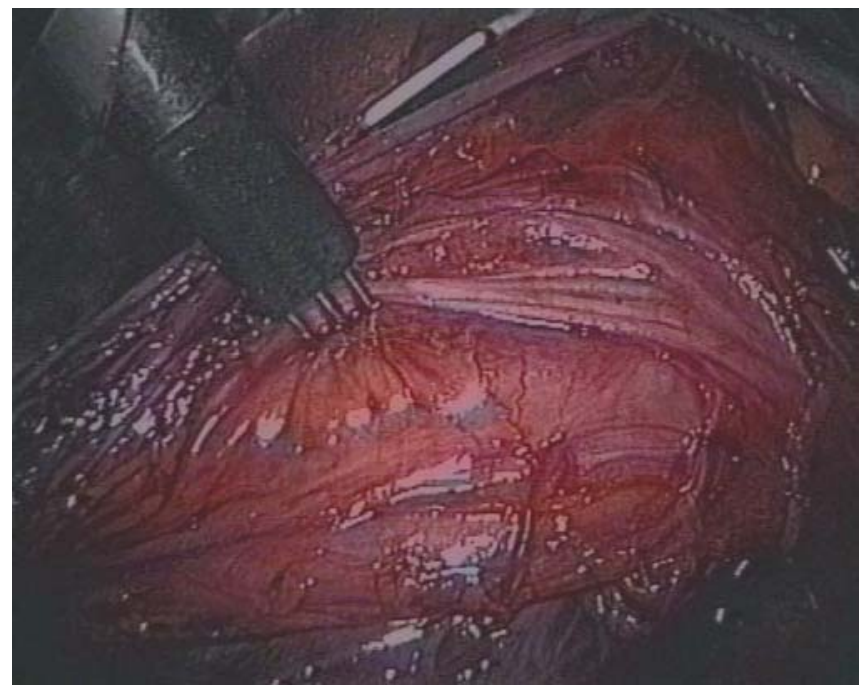

Fig. 1 Neurostimulation of the superior hypogastric plexus

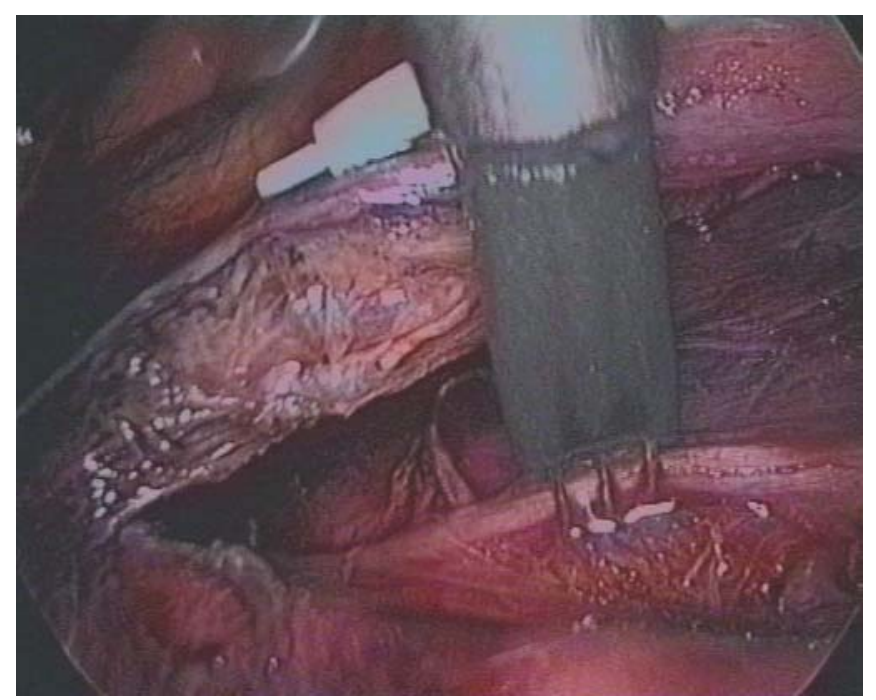

Fig. 2 Neurostimulation of the left sympathetic trunk

nerves did not result in any measurable reaction in the EMG reading under the same conditions. Therefore, stimulation was performed without any muscle relaxant.

\section{Electrostimulation of the autonomous nerves}

By electrostimulation of the superior hypogastric plexus and the two sympathetic trunks, no major changes in the vesical or rectal pressure were observed (Figs. 1, 2). By stimulation of the sympathetic trunk, a decrease in the central blood pressure between 10 and $30 \mathrm{mmHg}$ was observed. By stimulation of the splanchnic pelvic nerves we could show an increase in both intravesical and intrarectal pressure. This increase of rectal pressure was obtained by stimulation of the more medial and dorsal splanchnic nerves, while stimulation of the more ventral 


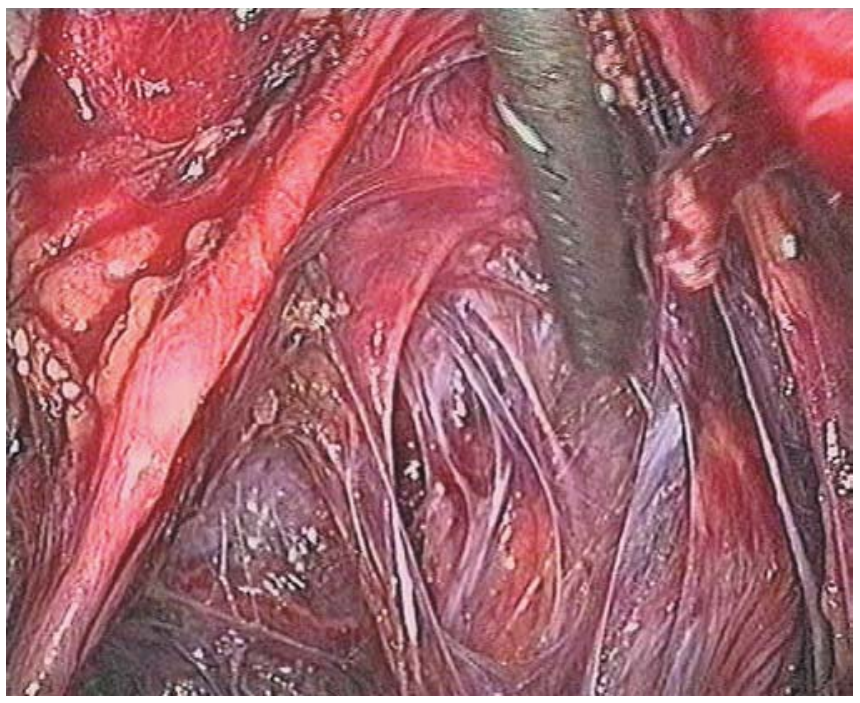

Fig. 3 Exposure of the pelvic splanchnic nerves

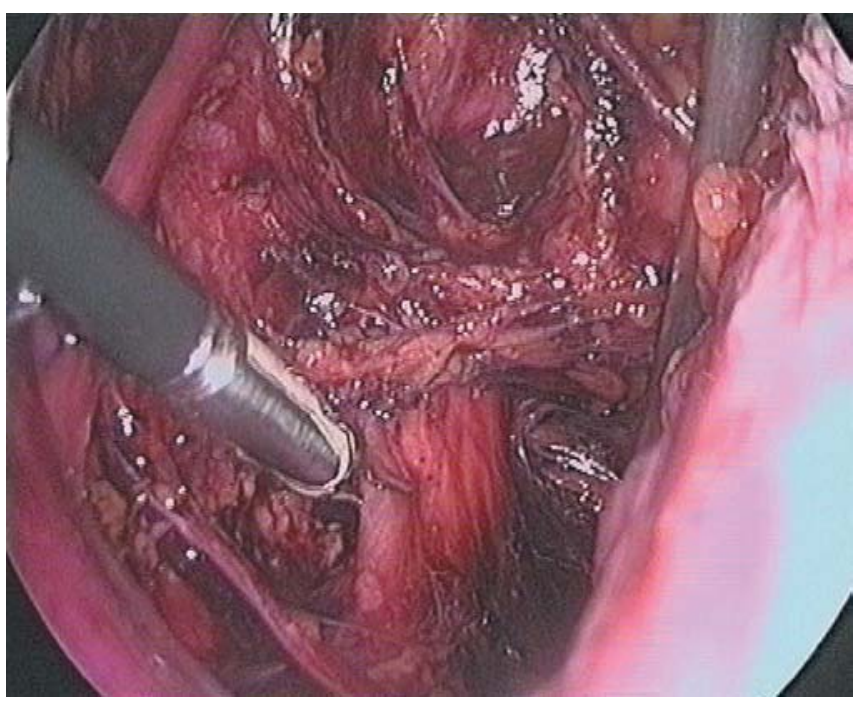

Fig. 4 Neurostimulation of the left sciatic nerve

and lateral nerves caused more changes to the intravesical pressure.

\section{Electrostimulation of the somatic nerves}

The identification and dissection of the femoral nerve, the sciatic nerve and the superior gluteus nerve were obtained in all patients. By electrostimulation of these motor nerves, we did not observe any fasciculation or anarchic contraction of muscle or muscle parts, but we observed specific and harmonious muscular contraction, harmonious movement of a part of the lower limb or the gluteal region: By electrostimulation of the femoral nerve, contraction of different muscles of the ventral part of the thigh will result in an extension of the knee (Figs. 3, 4). Elective electrostimulation of the gluteal nerves results in contraction of both gluteal nerves, resulting in extension of the thigh. Concerning neurostimulation of the sciatic nerve, we found a correlation between the part of the nerve that was stimulated and the level of the contracted muscle: The more caudally the sciatic nerve was stimulated, the more caudal was the level of the muscle response. Thus, stimulation of the caudal border of the sciatic nerve resulted in a plantar flexion/extension. By using magnification on the endoscope, neurolysis with elective electrostimulation of different parts of the sciatic nerve or of the femoral nerve was feasible.

\section{Discussion}

The pelvic wall and the parametric areas are important neuroanatomic areas for pelvic surgeons to understand because significant morbidity can ensue when suture or coagulation of nerves is performed. Due to the magnification effect of the endoscope and the cleanliness of the surgical preparation, during laparoscopy the surgeon gains a better view of the pelvis and may be able to recognize "new neural structures." Using laparoscopic neurostimulation, the surgeon may be able to gain intraoperative information about the function of these nerves. Based on this knowledge, we have developed nerve-sparing techniques for radical surgery of deep infiltrating endometriosis of the rectovaginal space [5].

While the topographic anatomy of different groups of autonomic nerves at their origin is clear, their topographic distribution in the depth of the pelvis-especially in the inferior hypogastric plexus-is poorly understood. By laparoscopic neurostimulation we have found that the inferior hypogastric plexus is not an anarchic patchwork of nerves, but presents an organization of the different kinds of nerves: (1) The cranial part of the inferior hypogastric plexus - the s.c. "sacrouterin ligament"-is composed of the sympathetic fibers coming from both inferior hypogastric nerves, which are responsible for the pain sensation of the cervix, and the upper part of the vagina. (2) The sympathetic fibers coming dorsally from the two sympathetic trunks anastomize with the inferior hypogastric plexus in the middle part of the supralevator portion of the rectal pillar. These nerves are responsible for the sensation of fullness of the bladder and rectum. (3) The parasympathetic nerves - the splanchnic pelvic nerves-anastomize with the inferior hypogastric plexus more laterally and caudally. The medio-dorsal fibers are responsible for the contraction of the terminal rectum, while the more latero-ventral fibers control bladder contraction.

This new knowledge of the topographic functional anatomy of the pelvic nerves should not only have implications for pelvic surgery, but should also have capital therapeutic implications for paraplegic patients. At present the only realistic approach to restore locomotion in paralyzed patients is the functional electrostimulation of infralesional nerves by implanted neuroprothesis [6]. Previous techniques with computer-aided locomotion by 
implanted electrical stimulation have been described elsewhere $[7,8,9]$, but by applying these techniques, epimysial electrodes were implanted. Their implantation required a traumatic surgical approach particularly for the gluteal nerves and, because of muscle contraction, fibrosis between the electrode and the muscle occurred after a short time resulting in less electrical transmission. Thus, at the moment, neural electrodes are preferred, but have to be placed directly on the nerves. In our work, we were able to expose all the different nerves of the pelvis, and implantation of the neuroelectrodes in this way is now technically possible. We have a minimalist approach to all somatic nerves implicated in standing/locomotion at their origin in the pelvis, where practically no motion between the nerves and the perineural tissue occurred.

Moreover, although recovery of locomotion will increase the quality of life and the freeness of paraplegic patients, vesical and renal function will determine the long-term prognosis of these paraplegic patients. Thus, for neurological dysfunction of the bladder or rectum, sacral root stimulation with posterior rhizotomy was performed using a dorsal approach [10]. During the same laparoscopic approach for the implantation of neuroelectrodes to the somatic nerves, elective implantation of neuroelectrodes at the rectal and vesical splanchnic nerves with elective transsection of the sympathetic fibers is now technically possible also, and new ways of improving defecation and miction in paralyzed patients should be researched. If the surgeon does not know exactly what type of nerves he has to expose or where he has to implant the electrodes, laparoscopic neurostimulation with recto-urodynamic recording should help in making this decision. Likewise, superior hypogastric plexus stimulator implantation to obtain semen from parapelgic men [11] has been performed by laparotomy and could now be performed by laparoscopy.
Our data provide strong evidence that a laparoscopic approach to the examination of pelvic nerves and a better understanding of their function may open new interdisciplinary approaches in pelvic surgery and in the management of para/tetraplegic patients.

\section{References}

1. Possover $\mathrm{M}$ et al (2000) Identification and preservation of the motoric innervation of the bladder in radical hysterctomy type III or IV. Gynecol Oncol 79:154-157

2. Ranade RG, Damale UB (1991) Radical surgery for cervical carcinoma: experience with "the Tokyo method." India J Cancer 28:99-100

3. Höckel M et al (1998) Liposuction-assisted nerve-sparing extended radical hysterectomy: oncologic rationale, surgical anatomy, and feasibility study. Am J Obstet Gynecol 178:971975

4. Possover M et al (1998) Laparoscopic para-aortic and pelvic lymphadenectomy: experience with 150 patients and review of the literature. Gynecol Oncol 71:19-28

5. Possover $\mathrm{M}$ et al (2000) Laparoscopic-assisted vaginal resection of recto-vaginal endometriosis. Obstet Gynecol 96:304307

6. Rabischong P (1999) Modern techniques of restoration of locomotion in paralyzed persons. Bull Acad Natl Med 183:531-537

7. Doyle J et al (1992) Augmentation of the accuracy percutaneous electrode implantation using a modified arthroscope to guide insertion. Arthroscopy 8:162-165

8. Meadows P et al (1993) Multichannel implantable electrical stimulation system for gait assist and excercise in the stroke and SCI popolation. 1993 Proceedings of the Ljubljana FES Conference (Ljubljana, Slovenia), pp 38-41

9. Peckham PH (1987b) Functional electrical stimulation: current status and future prospects of applications to the neuromuscular system in spinal cord injury. Paraplegia 25:274-288

10. Brindley GS (1994) The first 500 sacral anterior root stimulators: general description. Paraplegia 32:795-805

11. Brindley GS, Sauerwein D, Hendry WH (1989) Hypogastric plexus stimulators for obtaining semen from paraplegic men. $\mathrm{Br}$ J Urol 64:72-77 